\title{
Study on the runout of granular columns with SPH methods
}

\author{
Xuzhen He \\ Department of Engineering, University of Cambridge \\ Cambridge, United Kingdom \\ Dongfang Liang \\ Department of Engineering, University of Cambridge \\ Cambridge, United Kingdom
}

\begin{abstract}
Landslides are catastrophic geophysical phenomena, which may cause heavy fatality and property losses. Hence, it is of vital importance to understand their mechanisms and evaluate their travel distance, so that appropriate measures can be taken to mitigate their risk. This paper reports on an application of the incompressible Smoothed Particle Hydrodynamics (SPH) method to the simulation of the collapse of granular columns onto the planes of different slopes, which is similar to dry landslides. Numerical results show that the non-dimensional runout is a useful parameter in describing the travel distance as it depends only on the initial aspect ratio. Moreover, the traditional model with a constant friction angle is compared with the modified Mohr-Coulomb model with a variable friction angle sensitive to the shear-rate. It is found that traditional Mohr-Coulomb model with a fixed friction angle is incapable of always predicting the correct runout with different combinations of the aspect ratios and inclined angles. The shear-rate dependence effect must be considered for slim granular columns collapsing onto steep slopes. In addition, the taller granular columns travel much further than slowly released columns.
\end{abstract}

KEY WORDS: Landslides; granular flows; Mohr-Coulomb model; Smoothed Particle Hydrodynamics

\section{INTRODUCTION}

As the growth of population, mountainous areas have been more crowded, where many steep slopes are prone to landslides. When landslides happen, they can claim lives and threaten infrastructure. In addition, they often take place suddenly and move too fast for any mitigation measures to be enacted. Consequently, to quantitatively assess the risk of landslides and properly use the lands in mountainous cities, a proper method for predicting the propagation of landslides is necessary. In particular, the prediction of the travel distance of landslides has been a major task for many researchers

Some researchers predicted the travel distance through empiricalstatistical approaches. Unsurprisingly, these approaches found that travel distance depends, to some extent, on the volume of landslide mass (Corominas, 1996). As expected, the property of the sliding material also has an influence on the travel distance. Debris flows often show a larger mobility than landslides and rock falls. Furthermore, the dilatancy during failure process, the geometry of the slope and the degree of confinement of the travel path should also be taken into consideration when predicting the travel distance. One limitation of the empirical models is that they are only applicable to the landslides whose conditions are similar to those under which the models are derived. There are also dynamic models, which are physically based. These models generally consider the momentum and/or energy conservation of the flow. A widely-used continuum based dynamic model is developed by Savage and Hutter (1989). They incorporated the Mohr-Coulomb model with depth-averaged techniques to construct a set of equations for dry granular flows. As a relatively new computational technique, the Smoothed Particle Hydrodynamic (SPH) method has been increasingly used for developing the dynamic models.

The SPH method is a particle-based Lagrangian method (Monaghan, 2012), where the continuum is represented by a large number of discrete particles. SPH was initially used in astrophysics and fluid dynamics (Monaghan, 2012). Efforts have also been made in implementing Mohr-Coulomb type models with the SPH method in studying the dynamic problems of soils (Bui \& Fukagawa, 2008; Bui et al, 2011; Liang \& He, 2014). In this paper, the Mohr-Coulomb type models are implemented into the SPH framework and employed for the study of landslide runout.

Direct observations and detailed measurements of the dynamics of insitu landslides are scarce. What is more, these historical landslide data are not reproducible, which reduces their reliability. However, smallscale laboratory experiments of granular materials collapsing onto horizontal and inclined plates are reproducible and easy to conduct. These collapsing phenomena share some similarities with the propagation of landslides. Therefore, these controlled small-scale experiments are used in this paper as benchmarks.

\section{COLLAPSES OF GRANULAR COLUMNS}

Recently, a number of studies have been presented about the transient granular flows formed by the sudden release of vertical columns of grains onto horizontal or inclined planes. As in landslides, these granular flows can be modeled by the Mohr-Coulomb type models. 
Both collapses and landslides involve unsteady flows and their free surfaces change with time greatly. If the inclined slope angle is smaller than a certain value, the moving body would finally deposit on the slope and this process is characterized by the continuous deposition of grains on top of the already stationary grains.

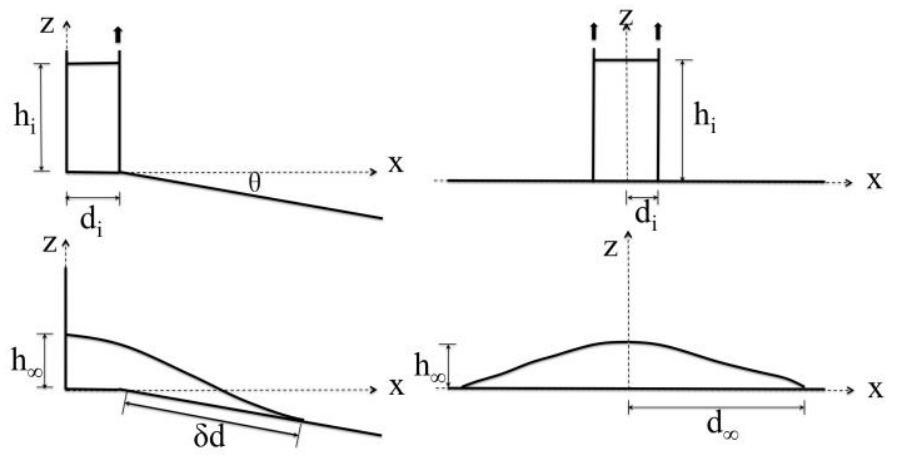

(a)

(b)

Fig. 1 Sketch of the experiment conditions and variables.

These granular flow experiments include two-dimensional unidirectional collapses and symmetrical bidirectional collapses in a channel of constant width (Balmforth \& Kerswell, 2005; Lube et al, 2011, 2005). Fig. 1 gives a sketch of the experiment conditions and the defined variables. The channel width is in the direction perpendicular to the page and is often very large. Granular columns are initially contained in a rectangular box. The initial width and height of the columns are denoted as $d_{i}$ and $h_{i}$ respectively. For the symmetrical collapses, the initial width $d_{i}$ is defined as half of the width of the box. The aspect ratio is $=h_{i} / d_{i}$. There are removable gates on the sides where collapses happen. Connected to the removable gates are horizontal or inclined channels with an inclined angle denoted by $\theta$. After the gate is suddenly removed, the granular column will collapse onto the slope. If the slope is mild, the granular materials will finally deposit onto the slope. With regard to the final deposit profile, the runout $(d)$ is the distance that the front has travelled along the slope, and the final spread of the profile is defined as $d=d+d_{i} / \cos$. In the experiments included in this study, columns were released in chutes whose width between two sidewalls is larger than the characteristic height $\left(h_{i}\right)$, width $\left(d_{i}\right)$ of granular columns. Therefore, the friction of sidewalls is negligible and these experiments can be straightforwardly regarded to be two-dimensional.

In this study, we are interested in studying how the characteristic height $\left(h_{i}\right)$, width $\left(d_{i}\right)$ and the slope angle $(\quad)$ influence the runout of granular materials.

\section{PHYSICAL MODEL FOR GRANULAR COLLAPSES}

The mobilisation of granular materials has been extensively researched in the soil mechanics community. The Mohr-Coulomb type models are commonly used in modeling the movement of soils and rocks. For the flow of granular materials, Savage \& Hutter (1989) developed a set of reduced equations with the Mohr-Coulomb model that faithfully reproduces the dynamic of dry granular avalanches. Similarly, Mohr-Coulomb type model can be incorporated into SPH method to study the collapse of granular columns. The conventional Mohr-Coulomb model for cohesionless materials reads $\tau_{i j}=\mu_{s} P \frac{\dot{\gamma}_{i j}}{|\dot{\gamma}|}$

where ${ }_{i j}$ is the deviator stress tensor, ${ }_{s}$ is the friction coefficient and $=\tan$, where is the friction angle. $\dot{\gamma}_{i j}$ is the shear rate tensor and $|\dot{\gamma}|$ is the second invariant of shear rate tenor $\dot{\gamma}_{i j}$, and $P$ is the local pressure.

The shear-rate dependent characteristic of the Mohr-Coulomb friction angle is generally not considered in the existing models for predicting dry granular avalanches, hence the influence of this effect on the runout has not been well understood. The Mohr-Coulomb model originates from the observation of shearing experiments under the quasi-static regime. In this regime, the inertial effects are negligible, and the contact forces between grains are balanced by the gravity force. Nevertheless, in rapid landslides and granular avalanches, some grains are highly agitated, and grains interact with each other via frequent binary collisions instead of enduring contacts, which is termed as collisional regime by most researchers (Campbell 2006). It has been proved by both physical experiments and discrete element simulations that the Mohr-Coulomb friction angle in the inertial regime and the collisional regime becomes larger (Midi 2004). Jop et al. (2006) developed a model to account for the shear-rate dependent feature of the friction coefficient and demonstrated that his model successfully predicted the three-dimensional granular flow in a chute experiment with two rough walls. The shear-rate dependent MohrCoulomb model can be expressed as

$\tau_{i j}=\left(\mu_{s}+\left(\mu_{\infty}-\mu_{s}\right) \frac{1}{I_{0} / I+1}\right) P \frac{\dot{\gamma}_{i j}}{|\dot{\gamma}|}$

where ${ }_{s}=\tan { }_{s}, \quad=\tan$ and $I_{0}$ are model parameters. $I$ is the inertial number calculated by

$I=\frac{|\dot{\gamma}| d_{s}}{\sqrt{P / \rho_{s}}}$

with ${ }_{s}$ being the density of grains and $d_{s}$ the average diameter of grains. The effective friction coefficient can therefore be defined as

$$
e=\left({ }_{s}+\left(\begin{array}{ll}
\infty & s
\end{array}\right) \frac{1}{I_{0} / I+1}\right)
$$

As can be seen, when the shear rate $|\dot{\gamma}|$ is zero, then the inertial number is also zero and the effective friction coefficient is ${ }_{s}$. Therefore, ${ }_{s}$ is called the quasi-static friction coefficient. When the shear rate $|\dot{\gamma}|$ is very large and the inertial number approaches infinity, then the effective friction coefficient is . Hence, is called the ultimate friction coefficient. In the shear-rate dependent MohrCoulomb model, the effective friction coefficient increases with the increase of shear rate. In contrast, the effective friction coefficient for the conventional Mohr-Coulomb model is constant.

Since the granular columns are prepared in a loose state, the volume change in initial failure process is negligible and the variation of void ratio during the propagation process is also relatively small based on experimental observations (Forterre \& Pouliquen, 2008; Savage \& Hutter, 1989). Therefore, an incompressible assumption is made.

\section{SMOOTHED PARTICLE HYDRODYNAMICS}

In smoothed particle hydrodynamics, the evaluation of variables and 
differentiation of variables can be carried out through the sum operations over neighboring particles with the help of kernel approximation and particle approximation. For example, for a variable $f(\mathbf{x})$, the SPH estimate at position $\mathbf{x}_{a}$ can be expressed as

$$
\left\langle f\left(\mathbf{x}_{a}\right)\right\rangle={\stackrel{m}{b=1}{ }_{b}}_{b} f\left(\mathbf{x}_{b}\right) W\left(\left|\mathbf{x}_{a} \quad \mathbf{x}_{b}\right|, h\right)
$$

The SPH estimate of $\nabla f(\mathbf{x})$ at position $\mathbf{x}_{a}$ can be calculated as

$$
\left\langle\nabla f\left(\mathbf{x}_{a}\right)\right\rangle=\sum_{b=1}^{N} \frac{m_{b}}{b} f\left(\mathbf{x}_{b}\right) \nabla W\left(\left|\mathbf{x}_{a} \quad \mathbf{x}_{b}\right|, h\right)
$$

where $m_{b}$ is the mass of the particle positioned at $\mathbf{x}_{b}$, and ${ }_{b}$ is its density. On the right hand sides of these equations, the sum is over all neighboring particles and $W\left(\begin{array}{ll}\mathbf{x}_{a} & \mathbf{x}_{b}\end{array} \mid, h\right)$ is the smoothing function.

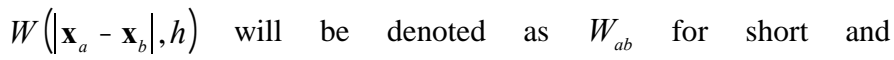

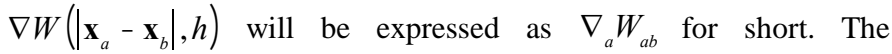
smoothing function is commonly an even function satisfying the renormalization condition and compact condition. In this study, the 2D cubic spline kernel is used. In addition, we also employed the corrected kernel gradient, whose usage has been proven to lead to improved accuracy of the SPH estimation (Liu \& Liu, 2010).

$\nabla_{a} \tilde{W}_{a b}=\mathbf{L}_{a} \nabla_{a} W_{a b}$

where the correction matrix $\mathbf{L}_{a}$ is defined as

$$
\mathbf{L}_{a}=\left(\sum_{b=1}^{N} \frac{m_{b}}{b} \nabla_{a} W_{a b} \otimes\left(\begin{array}{ll}
\mathbf{r}_{b} & \mathbf{r}_{a}
\end{array}\right)\right)^{1}
$$

From now on, the bracket representing the SPH estimate of a variable will be dropped to avoid clutter in the expressions.

\section{Incompressible SPH Implement}

Conservation of mass and momentum in Lagranrian form reads

$$
\begin{aligned}
& \frac{D}{D t}=\nabla \cdot \mathbf{v} \\
& \frac{D \mathbf{v}}{D t}=\mathbf{g} \quad \frac{1}{-} \nabla p+\frac{1}{-} \nabla .
\end{aligned}
$$

An incompressible SPH technique is used to solve the two equations above, in which the pressure projection (Cummins \& Rudman, 1999) method is implemented to impose the incompressibility constraint. The technique consists of a predictor step and a corrector step (Shao \& Lo, 2003).

The first step is the integration in time considering only the gravity term and shear stress term in the momentum equation. The intermediate velocity and position of the particle $a$ are obtained as follows

$$
\begin{aligned}
& \mathbf{v}_{a, 1}=\left(\mathbf{g}+\frac{1}{a} \nabla \cdot{ }_{a}^{t}\right) t \\
& \mathbf{v}_{a}=\mathbf{v}_{a}^{t}+\mathbf{v}_{a, 1} \\
& \mathbf{x}_{a}=\mathbf{x}_{a}^{t}+\frac{1}{2}\left(\mathbf{v}_{a}^{t}+\mathbf{v}_{a}\right) t
\end{aligned}
$$

Here $t$ is the time step. For the particle $a, \mathbf{v}_{a}$ is the intermediate velocity, $\mathbf{x}_{a}$ is the intermediate position, $\mathbf{v}_{a}^{t}, \mathbf{x}_{a}^{t}$ and ${ }_{a}^{t}$ are the velocity, position and shear stress tensor, respectively, at the previous time step. The SPH formulation of equation (11) is

$$
\Delta \mathbf{v}_{a, 1}=\left(\mathbf{g}+\sum_{b=1}^{N} m_{b}\left(\frac{\tau_{b}^{t}}{\rho_{b}^{2}}+\frac{\tau_{a}^{t}}{\rho_{a}^{2}}\right) \cdot \nabla_{a} \tilde{W}_{a b}\right) \Delta t
$$

Khayyer et al. (2009) showed that the usage of the corrected kernel gradient not only ensures the exact calculation of any linear momentum but also guarantees the conservation of angular momentum in updating equation (14). Such numerical treatment has been implemented to improve the accuracy of SPH calculation.

In the predictor step, incompressibility is not enforced. Then, a corrector step is introduced to adjust the intermediate density to its original value. The correction to the velocity is obtained by considering the pressure term

$$
\mathbf{v}_{a, 2}=\left(\frac{1}{a} \nabla p_{a}^{t+1}\right) t
$$

Its $\mathrm{SPH}$ formulation is

$$
\Delta \mathbf{v}_{a, 2}=-\sum_{b=1}^{N} m_{b}\left(\frac{p_{b}^{t+1}}{\rho_{b}^{2}}+\frac{p_{a}^{t+1}}{\rho_{a}^{2}}\right) \nabla_{a} \tilde{W}_{a b} \Delta t
$$

In this paper, we employed the divergence of intermediate velocity to calculate the change of density in the predictor step such that

$\frac{1}{\rho_{a}} \sum_{b=1}^{n} m_{b} \nabla_{a} \tilde{W}_{a b}^{*} \cdot\left(\mathbf{v}_{a}^{*}-\mathbf{v}_{b}^{*}\right)+\nabla \cdot\left(\Delta \mathbf{v}_{a, 2}\right)=0$

It is shown that this equation has higher order than traditional source term and can improve the calculated pressure field (Khayyer et al., 2009).

Combining equation (13) and equation (15) yields the pressure Poisson equation

$$
\nabla \cdot\left(\frac{1}{\rho_{a}^{*}} \nabla p_{a}^{t+1}\right)=\frac{1}{\rho_{a} \Delta t} \sum_{b=1}^{n} m_{b} \nabla \tilde{W}_{a b}^{*} \cdot\left(\mathbf{v}_{a}^{*}-\mathbf{v}_{b}^{*}\right)
$$

It is possible to construct the SPH Laplace operator by employing equation (6) twice. However, it is reported that this direct projection is sensitive to boundary conditions and often causes instability; we use an approximate projection that is analogous to the approximation of the viscous diffusion term in many researches. The chosen SPH formulation of the Laplace operator is

$$
\nabla \cdot\left(\frac{1}{a} \nabla p_{a}^{t+1}\right)=\sum_{b=1}^{N} m_{b} \frac{8}{\left({ }_{a}+{ }_{b}\right)^{2}}\left(\begin{array}{ll}
p_{a}^{t+1} & p_{b}^{t+1}
\end{array}\right) \frac{\mathbf{x}_{a b} \cdot \nabla_{a} W_{a b}}{\left|\mathbf{x}_{a b}\right|^{2}+{ }^{2}}
$$

where $\quad \mathbf{x}_{a b}=\mathbf{x}_{a} \quad \mathbf{x}_{b}$ and is a small value to always keep the denominator non-zero. Therefore, after the predictor step, this Poisson equation is solved based on the intermediate density to obtain the new pressure.

Finally, the velocity and position at next time step is updated by

$$
\begin{aligned}
& \mathbf{v}_{a}^{t+1}=\mathbf{v}_{a}+\mathbf{v}_{a, 2}=\mathbf{v}_{a}^{t}+\mathbf{v}_{a, 1}+\mathbf{v}_{a, 2} \\
& \mathbf{x}_{a}^{t+1}=\mathbf{x}_{a}^{t}+\frac{1}{2}\left(\mathbf{v}_{a}^{t}+\mathbf{v}_{a}^{t+1}\right) t
\end{aligned}
$$

\section{Implement of the Mohr-Coulomb Type Model}

The Mohr-Coulomb type equations are implemented in the incompressible SPH model in the predictor step. At first, the local shear rate tensor of every particle is calculated. Based on the definition of shear rate tensor

$\dot{\gamma}_{a}^{t}=\left(\nabla \mathbf{v}^{t}+\left(\nabla \mathbf{v}^{t}\right)^{T}\right)_{a}$ 
Its $\mathrm{SPH}$ formulation is obtained

$\dot{\gamma}_{a}^{t}=\sum_{b=1}^{N} \frac{m_{b}}{\rho_{b}}\left[\left(\mathbf{v}_{b}^{t}-\mathbf{v}_{a}^{t}\right) \otimes \nabla_{a} \tilde{W}_{a b}+\nabla_{a} \tilde{W}_{a b} \otimes\left(\mathbf{v}_{b}^{t}-\mathbf{v}_{a}^{t}\right)\right]$

Then, the shear rate $|\dot{\gamma}|$ is calculated from the shear rate tensor. Then, equation (1) (2) are used to estimate local shear stress for every particle.

\section{Boundary Conditions}

When updating the velocity and position of particles using equations (14) and (16), no special treatment is needed for free surface particles. For wall boundaries, the dummy particle method is adopted. For free surface particles, the pressure is assigned to zero. In the ISPH method, a particle is labeled as a free surface particle if the density calculated at the particle satisfies the following condition:

${ }_{a}<0$

where $\beta=0.99$ and ${ }_{0}$ is the constant initial density.

\section{APPLICATION TO COLLAPSES OF GRANULAR COLLUMNS}

In the experiments (Lube et al, 2011, 2005), four kinds of granular materials (Coarse quartz sand, fine quartz sand, rice and sugar) were used. These materials have different average diameters, but they produce the same flow process and the same final deposition shape as they have almost identical quasi-static friction angle, whose value is $31^{\circ}$ as is provided by the researchers. Two additional parameters are needed for shear-rate dependent Mohr-Coulomb model. As reported in Jop et al. (2006) and Midi (2004), the ultimate friction angle is generally $10^{\circ}$ larger than the quasi-static friction angle. In the ratedependent model, $I_{0}$ is chosen based on a trial and error procedure such that the effective friction angle in static region is close to quasistatic friction angle and it is relative large for the violent flowing region. In all the simulations, the fluid particles are initially arranged in a regular square lattice, with boundary particles added to form the wall and bed.
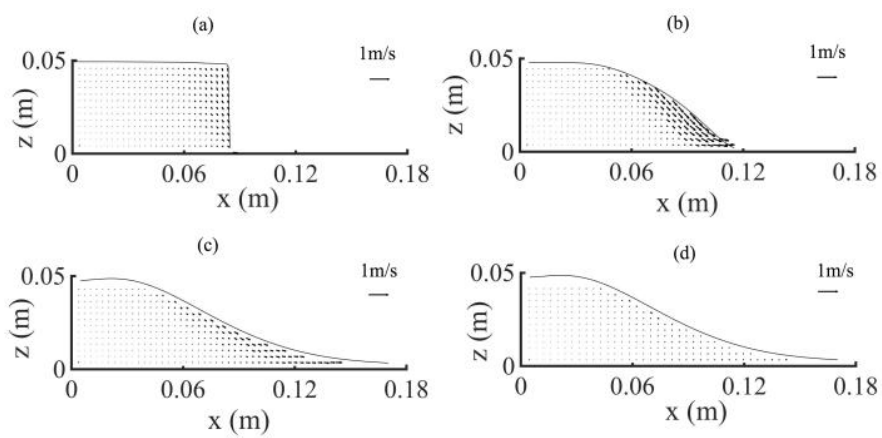

Fig. 2 Evolution of velocity for the collapse of a shallow column, $h_{i}=0.05 \mathrm{~m}, d_{i}=0.083 \mathrm{~m}$; (a) $0.02 \mathrm{~s}$ (b) $0.1 \mathrm{~s}$ (c) $0.2 \mathrm{~s}$ (d) $0.4 \mathrm{~s}$.

\section{Time Evolution of the Collapses}

Qualitative observations (Lube et al. 2005) in the experiments reveal a strong dependence of the flow behaviour on the value of the initial aspect ratio. The SPH simulations can reproduce the flow phenomena of different aspect ratios faithfully. For shallow piles (Fig. 2), only grains above a fracture surface participate in the flow at the beginning, as seen in Figure 2(a). This surface intersects the top of the column to form a straight line normal to the $\mathrm{x}-\mathrm{z}$ plane. When the flow continues, this line moves toward the vertical wall on the left, and more grains are eroded and get involved in the flow. For shallow piles, grains would come to a stop completely before this line reaches the left vertical wall. Therefore, for shallow columns, the height of the final profile is equal to the initial height $\left(h=h_{i}\right.$ ). The collapse of tall columns involves violent cascading movement (Fig. 3). There is a critical height, above which the grains move like a free fall at first, as seen in Figure 3(a). The grains between the critical height and the dynamic interface move downwards as well as laterally to the right. The eventual deposition takes the shape of a wedge and the final height is much smaller than the initial height.

An important feature of the collapse is the evolution of a dynamic interface that separates the static region from the flowing region. Initially, the interface appears to be a straight line intercepting the gate at the bottom corner, which is inclined at $61^{\circ}$ to the horizontal axis (Fig. 2(a) and Fig. 3(a)). By comparing these interfaces at different times (Fig. 2 and Fig. 3), we can conclude that the static region is growing with time and the interface evolves toward the free surface. When the interface coincides with the free surface, the granular flow comes to a stop completely.
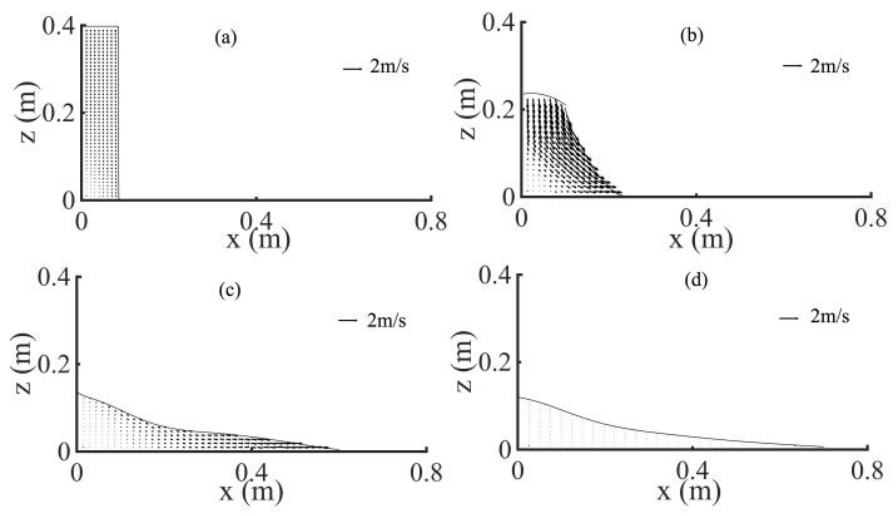

Fig. 3 Evolution of velocity for the collapse of a tall column, $h_{i}=0.415 \mathrm{~m}, d_{i}=0.083 \mathrm{~m}$; (a) $0.02 \mathrm{~s}$ (b) $0.2 \mathrm{~s}$ (c) $0.5 \mathrm{~s} \mathrm{(d)} 1.0 \mathrm{~s}$.

\section{Runout of Granular Columns}

The granular columns with larger volumes will have longer runout. Fig 4 shows the deposited profiles for the symmetric collapses of four different columns onto a horizontal plane. Only half of the finial profiles needs to be shown because of the symmetry. The conventional Mohr-Coulomb model is used with a $31^{\circ}$ friction angle. Two of these columns shown in Fig. 4 are prepared with the same aspect ratio $(\alpha=3)$ but with different cross-section areas. It can be seen that the column with a larger volume spreads further. However, the two final profiles are similar to each other. This indicates that the non-dimensional runout $\left(d / d_{i}\right)$ will be largely independent of the column volume.

The initial aspect ratio is an important parameter that influences the runout of granular collapses. Three of these columns are prepared with the same initial cross-section areas but with different aspect ratios $(\alpha=3,4$ and 5). It can be seen that, the larger the aspect ratio, the longer the runout, whereas the smaller the final height. This is the result of the higher initial potential energy associated with a taller column. 
Experiments show unique relationships between the non-dimensional runout $\left(d / d_{i}\right)$ and the aspect ratio $\left(=h_{i} / d_{i}\right)$. Fig. 5 gives the experimental results of unidirectional collapses as well as some numerical results. The model parameters and simulation conditions are listed in Table 1. All experimental results fall onto one unique line irrespective of the volume of columns. Therefore, the non-dimensional runout $\left(d / d_{i}\right)$ is a useful parameter for describing the distances travelled by the granular columns and its value only depends on initial aspect ratio.

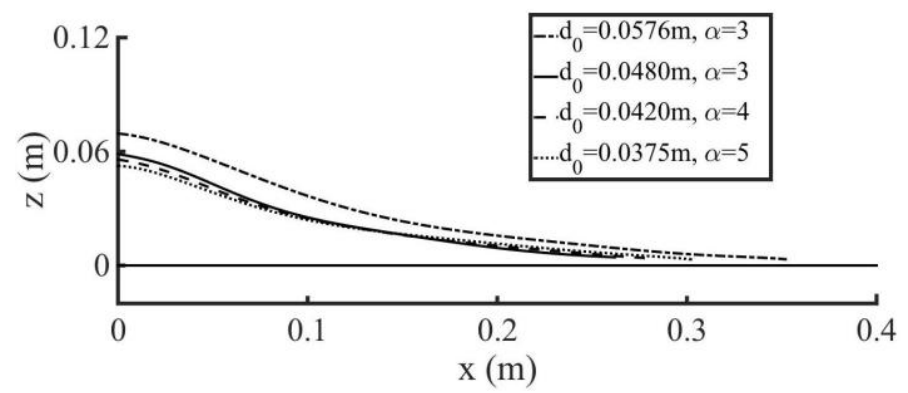

Fig. 4 Deposited profiles for symmetric collapses of four different columns onto horizontal bed.

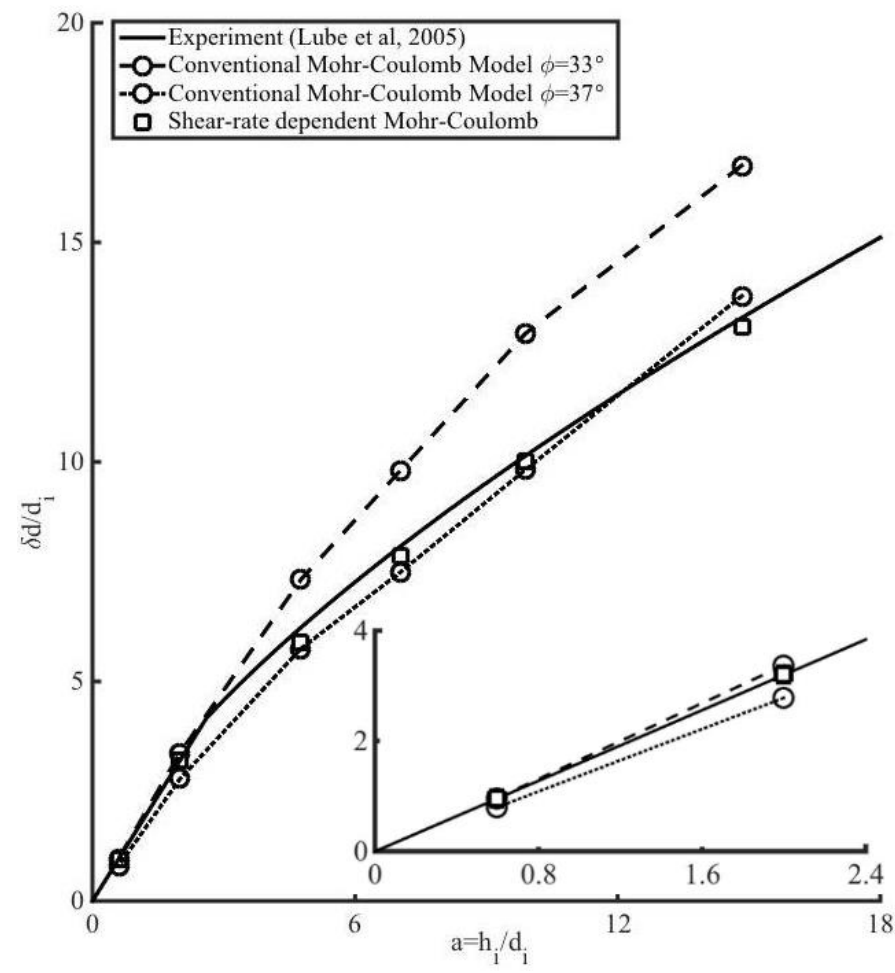

Fig. 5 Variation of non-dimensional final front position $\left(d / d_{i}\right)$ with aspect ratio $\left(h_{i} / d_{i}\right)$ for unidirectional collapses on horizontal bed.

It can be seen from Fig. 5 that conventional Mohr-Coulomb model cannot correctly capture the correct overall relation with a fixed friction angle. When a small friction angle $\left(33^{\circ}\right)$ is used, the predicted runout agrees well with experiments for aspect ratios below 3. For columns with aspect ratio higher than 3 , however, it significantly overestimates the runout. When a large friction angle $\left(37^{\circ}\right)$ is used, it still slightly overestimates the runout at $h_{i} / d_{i}=14.8$, at the same time it underestimates the runout of shallower columns. If back analysis is conducted to estimate the equivalent friction angle for the conventional Mohr-Coulomb model, a small value $\left(31^{\circ}-33^{\circ}\right)$ is needed for shallow columns and a value larger than $37^{\circ}$ is needed for columns with $h_{i} / d_{i}=14.8$. In contract, the shear-rate dependent Mohr-Coulomb model can predict the runout for columns with a large range of aspect ratios with only one set of parameters.

Table 1 Parameters for numerical tests of unidirectional collapses

\begin{tabular}{|c|c|c|c|c|}
\hline $\begin{array}{l}\text { Aspect } \\
\text { ratio }\end{array}$ & $\begin{array}{l}\text { Initial } \\
\text { height } \\
\text { (m) }\end{array}$ & $\begin{array}{l}\text { Numb } \\
\text { er of } \\
\text { SPH } \\
\text { fluid } \\
\text { particl } \\
\text { es }\end{array}$ & $\begin{array}{l}\text { Friction angle } \\
\text { for convectional } \\
\text { Mohr-Coulomb } \\
\text { Model }\end{array}$ & $\begin{array}{l}\text { Parameters for } \\
\text { Shear-rate } \\
\text { dependent } \\
\text { Mohr- } \\
\text { Coulomb } \\
\text { Model }\end{array}$ \\
\hline 0.596 & 0.0496 & 6448 & \multirow{6}{*}{$\begin{array}{r}=31^{\circ}, 33^{\circ}, \\
37^{\circ} \text { and } 41^{\circ}\end{array}$} & \multirow{6}{*}{$\begin{array}{c}s=31^{\circ}, \\
=41^{\circ}, \\
\frac{I_{0}}{\Gamma}=1\end{array}$} \\
\hline 1.999 & 0.1663 & 8192 & & \\
\hline 4.762 & 0.4 & 8400 & & \\
\hline 7.030 & 0.58 & 7656 & & \\
\hline 9.893 & 0.831 & 7756 & & \\
\hline 14.833 & 1.246 & 8544 & & \\
\hline
\end{tabular}

Some numerical tests are then conducted to simulate the unidirectional collapses on sloped beds of different inclined angles $\left(4.2^{\circ}, 10^{\circ}, 15^{\circ}\right.$, $20^{\circ}$ ) for a shallow column $\left(h_{i} / d_{i}=0.6\right)$ and for a tall column ( $\left.h_{i} / d_{i}=2\right)$. Figure 6 shows the relationship between the nondimensional final front position and the inclined angle of the slope. Experimental results are also included. The upper solid line is the results for the tall column and the lower solid line is for the shallow column. The value $31^{\circ}$ is chosen as the friction angle for the conventional Mohr-Coulomb model. The parameters for shear-rate dependent Mohr-Coulomb model are listed in Table 1. It is shown from the figure that, for the shallow collapse onto slopes less than $20^{\circ}$, the conventional Mohr-Coulomb model and shear-rate Mohr-Coulomb Model obtain similar results that agree equally well with experimental results. When the slope increases beyond $20^{\circ}$, the conventional MohrCoulomb model overestimates the front position by more than $40 \%$ of the experimental value. In contrast, the shear-rate dependent MohrCoulomb model gives only a $10 \%$ overestimate. For tall columns, similar results can be obtained, but the predicted results by these two models show clear discrepancy even when the slope is small. Although shear-rate dependent Mohr-Coulomb model obtains better result, it still slightly overestimates the front position by around $10 \%$ error on steep slopes, which can be attributed to the inaccuracy of parameters chosen.

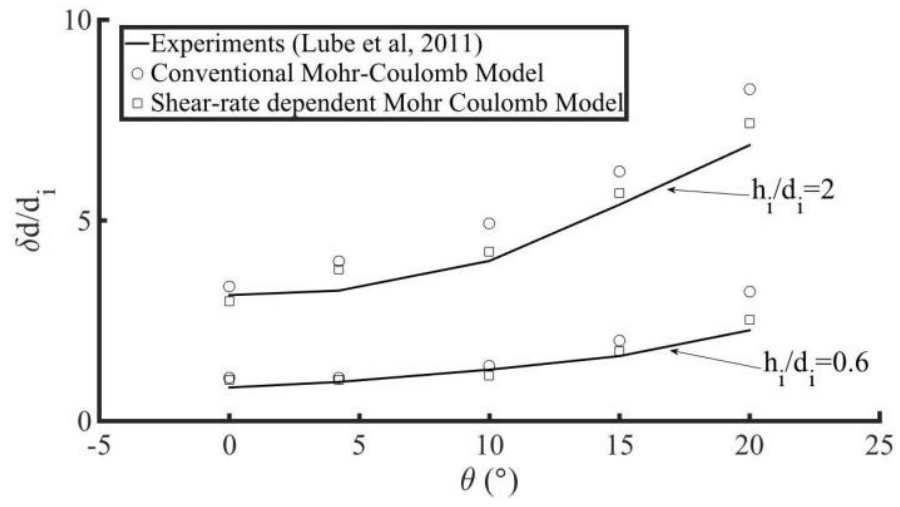


Figure 6 Variation of the non-dimensional final front position $\left(d / d_{i}\right)$ with the bed slope angle for unidirectional collapses.

The granular flow process consists of three stages, the mobilization stage, the flow stage, and the deposition stage. During the mobilization stage and deposition stage, the grains are in quasi-static regime and the effective friction coefficient is small. During the flow stage, grains are agitated and local shear rates are large enough to see an apparent increase in the effective friction coefficient. The results predicted by conventional Mohr-Coulomb model with a quasi-static friction angle overestimate the runout and the ceasing time. When the shallow columns are released onto gradual slopes, the total collapse time is small. Therefore, the duration of flow stage is relatively short and the influence of rate dependence effect is negligible. In contrast, when the columns are released onto steep slopes, the flow stage constitutes a significant part of the total process, and the influence of rate dependence is important. In addition, when the aspect ratio is larger, the depth of granular materials above the interface is larger and the static pressure at the interface is larger. As the shear stress depends on the local pressure in Mohr-Coulomb type models, the increased frictional angle caused by rate dependence effect is amplified by the large pressure. It is found that the rate dependence effect is negligible for the shallow granular flow along mild slopes. When the inclined floor steepens or the aspect ratio of granular column increases, the rate dependence effect becomes more prominent.

\section{Slowly Released Columns}

The final profiles for tall columns take on a wedge shape, which is similar to granular piles, formed by pouring granular materials slowly. Therefore, it is interesting to compare the runout made by suddenly collapsing and the runout made by slowly piling and study how the column inertia influences the runout. To avoid the sudden collapse, we release the column in several successive steps. At first, only part of a column is released onto the horizontal slope. After the first part reaches a static state, we release another bit of the column right on top of the already-deposited profile obtained from the previous step. This process continues until a certain volume of granular materials is totally released. Meanwhile, we increase the number of steps until convergent profile is obtained.

Table 2. Parameters for tests to find the slowly released pile

\begin{tabular}{|c|c|c|c|}
\hline & $d_{i}(\mathrm{~m})$ & $\begin{array}{c}\text { Released } \\
\text { column aspect } \\
\text { ratio for Step 1 }\end{array}$ & $\begin{array}{c}\text { Released column } \\
\text { aspect ratio for } \\
\text { Step 2 to } \mathrm{n}\end{array}$ \\
\hline 1 Column & 0.048 & 3 & 0 \\
\hline 2 Steps a & 0.048 & 2.3750 & 0.6250 \\
\hline 2 Steps b & 0.048 & 1.7188 & 1.2812 \\
\hline 5 Steps & 0.048 & 1 & 0.5 \\
\hline 10 Steps & 0.048 & 0.4688 & 0.2812 \\
\hline
\end{tabular}

Five sets of symmetrical tests are conducted to find the profile of slowly released pile for a column with an aspect ratio of 3 . Their parameters are listed in Table 2. Fig. 7 shows the final profiles obtained from SPH calculations for five tests. It can be seen that when we release the column for 5 or 10 steps, the profiles collapse onto each other, and convergence has been achieved. An important feature of the profile is that it has a uniform slope angle. In contrast, the final profile for the sudden collapse of a tall column has the same slope angle only at upper part of the profile, but the slope angle at the front is much smaller. Therefore, it can be concluded that a landslide travels further than the runout of slowly released piles, although the granular assembles in the two situations have the same volume.

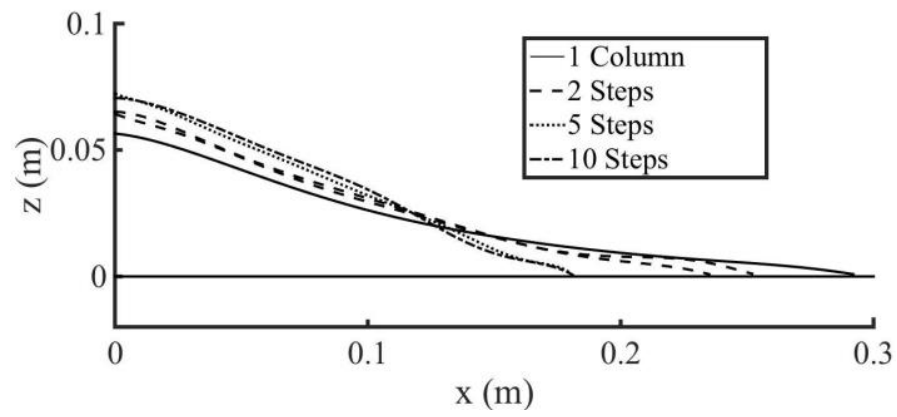

Fig. 7 Final profiles for five symmetric tests to find granular pile profile

If the slowly released granular pile has a uniform slope angle of $\theta_{s}$, then their theoretical relation between the runout and the aspect ratio is

$d / d_{i}=\sqrt{2 / \tan s} 1$

(25)

Fig. 8 compares the runout of sudden collapsing experiments and slowly released columns at different aspect ratios. Several SPH computational results are also included. It can be seen that for shallow columns, the collapsing runout is almost the same as the spreading of slowly released columns. However, when the aspect ratio increases, the discrepancy also increases. Therefore, the inertia of shallow columns is negligible and their runout can be predicted by the runout of slowly released columns. But, the inertia effect is significant in predicting the runout of tall columns.

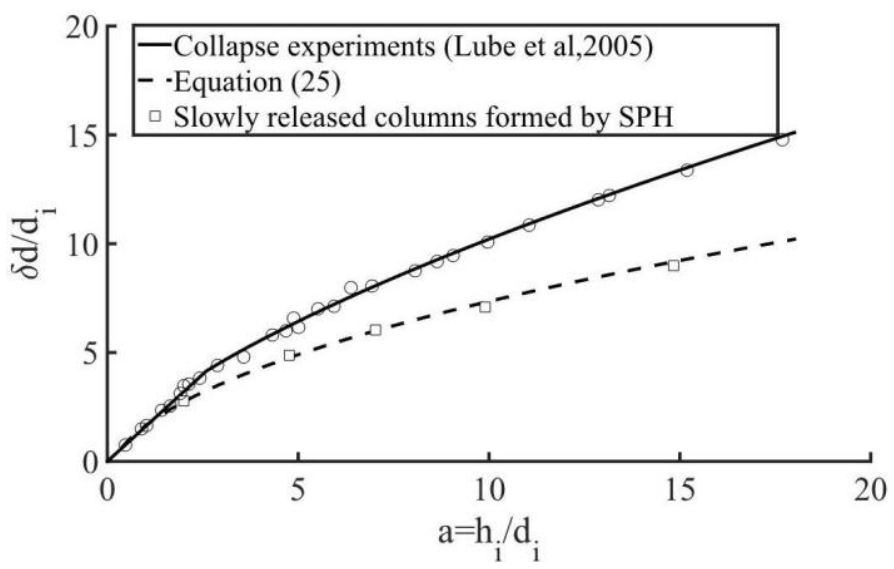

Fig. 8 Comparison of runout between sudden collapses and slowly released columns.

\section{CONCLUSION}

This paper presents the application of an incompressible SPH method to the prediction of runout of granular columns. In the incompressible SPH technique used, the shear rate tensor is evaluated by summing up the velocity information of neighboring particles. The local shear stress is calculated from the Mohr-Coulomb type equations.

This algorithm is verified against column collapse experiments first. All the key features can be successfully reproduced by the Mohr- 
Coulomb type models, such as the distinctive dependence of the flow behaviour on the initial aspect ratio of the column, the evolution a dynamic interface demarcating the static region and flowing region.

Then, the influence of different factors on the runout is investigated. It is found that the non-dimensional runout only depends on the initial aspect ratio and is independent on the volume. Conventional MohrCoulomb model with fixed friction angle cannot correctly predict the runout for a wide range of aspect ratios and inclined angles. The shear-rate dependence effect must be considered for tall granular columns collapsing onto steep slopes. The sudden collapse of a tall granular column produces a larger runout than the slow release of granular assembles of the same volume.

\section{ACKNOWLEDGEMENTS}

The research is supported by the National Natural Science Foundation of China (51479111) and the Non-profit Industry Financial Program of the Ministry of Water Resources (201401027). We also thank the Raymond and Helen Kwok Scholarship of Jesus College, University of Cambridge.

\section{REFERENCES}

Balmforth, NJ and Kerswell, RR (2005). "Granular collapse in two dimensions." Journal of Fluid Mechanics, 538(-1), 399.

Bonet, J and Lok, TSL (1999). "Variational and momentum preservation aspects of smooth particle hydrodynamic formulations." Computer Methods in Applied Mechanics and Engineering, 180(1-2), 97-115.

Bui, H and Fukagawa, R (2008). "Lagrangian meshfree particles method (SPH) for large deformation and failure flows of geomaterial using elastic-plastic soil constitutive model." International Journal for Numerical and Analytical Methods in Geomechanics, (February), 1537-1570.

Bui, HH, Fukagawa, R, Sako, K and Wells, JC (2011). "Slope stability analysis and discontinuous slope failure simulation by elasto-plastic smoothed particle hydrodynamics (SPH)". Géotechnique, 61(7), 565574.

Campbell, CS (2006). "Granular material flows - An overview." Powder Technology, 162(3), 208-229.

Corominas, J (1996). "The angle of reach as a mobility index for small and large landslides". Canadian Geotechnical Journal, 33, 260-271.

Cummins, SJ and Rudman, M (1999). "An SPH Projection Method." Journal of Computational Physics, 152(2), 584-607.

Forterre, Y and Pouliquen, O (2008). "Flows of Dense Granular Media." Annual Review of Fluid Mechanics, 40(1), 1-24.

Jop, P, Forterre, Y and Pouliquen, O (2006). "A constitutive law for dense granular flows." Nature, 441(7094), 727-30.

Khayyer, A, Gotoh, H, and Shao, S (2009). "Enhanced predictions of wave impact pressure by improved incompressible SPH methods." Applied Ocean Research, 31(2), 111-131.

Liang, D and He, X (2014). "A comparison of conventional and shearrate dependent Mohr-Coulomb models for simulating landslides." Journal of Mountain Science, 11(6), 1478-1490.

Liu, MB and Liu, GR (2010). "Smoothed Particle Hydrodynamics (SPH): an Overview and Recent Developments." Archives of Computational Methods in Engineering, 17(1), 25-76.

Lube, G, Huppert, HE, Sparks, RSJ and Freundt, A (2011). "Granular column collapses down rough, inclined channels." Journal of Fluid Mechanics, 675(2011), 347-368.

Lube, G, Huppert, H, Sparks, R, and Freundt, A (2005). "Collapses of two-dimensional granular columns." Physical Review E, 72(4), 1-10.

Midi, GDR (2004). "On dense granular flows." The European Physical Journal. E, Soft Matter, 14(4), 341-65.
Monaghan, JJ (2012). "Smoothed Particle Hydrodynamics and Its Diverse Applications." Annual Review of Fluid Mechanics, 44(1), 323-346.

Randles, P and Libersky, L (1996). "Smoothed particle hydrodynamics: some recent improvements and applications." Computer Methods in Applied Mechanics and Engineering, 7825(96).

Savage, S and Hutter, K (1989). "The motion of a finite mass of granular material down a rough incline." Journal of Fluid Mechanics, 199, $177-215$.

Shao, S, and Lo, EYM (2003). "Incompressible SPH method for simulating Newtonian and non-Newtonian flows with a free surface." Advances in Water Resources, 26(7), 787-800. 